\title{
Task-Driven: an Effort to Build Secondary Students Mathematical Reasoning Skills
}

\author{
E. Elvis Napitupulu \\ Mathematics Department \\ Universitas Negeri Medan \\ Medan, Indonesia \\ elvisnapit@gmail.com
}

\author{
Ani Minarni \\ Mathematics Department \\ Universitas Negeri Medan \\ Medan, Indonesia \\ Animinarni10@gmail.com
}

\begin{abstract}
It is already known widely that Indonesian students lack mathematical reasoning skills (MRS) at all level. Many efforts had been taken to overcome the problem. This study aimed to build upper secondary students MRS by means of disseminating an instructional materials, which had been proved valid, practice, and effective from the related previous research. At an amount of 136 tenth grade students from four public schools participated in the study. The students MRS average score for pre-test was 29.3 and 49 for post-test in 100-scale, and the normalized gain was 0.28 , which categorized low. Meanwhile, half of the students perceived that the tasks and the learning process lead and drove them to connect and make use of previous knowledge, achieve in-depth understanding, motivate higher, critical, and creative thinking, and enjoy solving problems together in groups.
\end{abstract}

Keywords-Task-driven; Mathematical reasoning skills

\section{INTRODUCTION}

Recalls on establishing mathematical reasoning ability in part of students had been launched since the last of eighties [1] and [2]. Both researchers and mathematics educators perceive the competency as one of the most important skill that students should master during their days in school. It plays central role not only in doing mathematics, but also for studying other disciplines, especially natural sciences. Actually, in all areas of disciplines, even in life, drawing conclusion is an anytime work. Particularly in mathematics, mathematical reasoning takes place while one needs to draw a conclusion.

Drawing conclusion occurs at any time in human's life. It serves providing alternatives to choose from which one determine what fits to him to do. It should be the best such that brings the most advantage, albeit it is not always the case. That is why the conclusion should be drawn correctly and feasible to execute. Generally, people draw a conclusion either in certainty or in probabilistic. In other words, deductively or inductively. The conclusion drawn deductively is the only one accepted and known to be valid, whereas the one which drawn inductively is treated as a conjecture, that needs a proof.

In doing mathematics, one always face a situation in which information or data is given. He then seeks or build a relationship between the data. He also possibly find a (some) consequence(s) from the information. The relationship or consequence he builds will then takes its role to continue the work to achieve the goal. He also often should apply some concepts, procedures, or principles he has in mind to the information in order to get an advanced conclusion. The case turns complex if the conclusion is possibly more than one. At this point, he can only do trial and error if not sure which one will run well. This process usually works repeatedly before arriving at the final target to reach. However, any step of the work needs mathematical reasoning.

The above paragraphs show that students are absolutely demanded to mastering mathematical reasoning skill. They only can make progress if they make use of their reasoning to either comprehend, connect among mathematical objects, represent, or communicate in the frame of problem solving. For this purpose, as an effort, teachers should facilitate the students working on tasks that force them not only imitate the worked examples provided in the classrooms but also think deeply, critically, and creatively to handle new and challenging problems. Concerning this, Lithner [3] emphasizes that students need a supporting environment in which they struggle grasping core mathematical competencies by means of solving mathematical problems. It is less evident that conventional teaching to be able to lead students achieving skills purposed.

Albeit the urgency of mastering the competency, Indonesian students at all levels showed underperformed MRS. TIMSS study [4] reported Indonesian eight graders only achieved $17 \%$ meanwhile international average was $30 \%$. Fourth graders only grasped $20 \%$, comparing to international average, which is $40 \%$ [5]. Recently, the previous works on Indonesian upper secondary students revealed the lack of the competency [6] and [7]. In [6] for example, they only scored $36.56 \%$, whereas in [7] they scored $37.96 \%$ averagely. It is important to note that they achieved these scores after engaging in a constructivist instruction. Before the intervention took place, they only scored 27.88 in 100 -scaled. It means that the intervention effected an improvement although categorized low. Therefore, it can be expected that the materials developed effect also to a wider subject. Based on these findings, the research then continued with different 
subjects. For this second phase of the research, the intervention still used the materials have been developed in the first phase with necessary modification and revision. This paper reported some part of the second phase of the research.

One of the responsible to the low achievement in MRS is hypothesized strongly due to the processes of teaching and learning conducted in Indonesian mathematics classrooms. Many researchers reported direct instruction still dominates Indonesian's mathematics classrooms [8], [9], and [10]. Teachers rarely give their students space and time to connect their previous knowledge to construct new ones. There did not exist any challenge to build conjecture and prove it, nor any challenge to explain mathematics idea or proposition. Students then are accustomed to receiving lessons passively. Furthermore, they only able to handle routine exercises similar to the ones their teachers gave.

Considering the fact, Indonesian Government via The Ministry of Education and Culture launched 2013-Curriculum, nowadays implemented in Indonesian schools. The new Curriculum appeals for adopting and using scientific approach to conduct mathematics lessons as part of models or approaches teachers use in classrooms. This approach, which constitutes of observing, questioning, experimenting, reasoning, and communicating, would enable students to develop their scientific skill especially in reasoning. To accelerate the dissemination of the teaching approach used in classrooms, the Indonesian Government then integrate it in Teacher Certification Program.

Conducting mathematics lessons based on scientific approach is one of many major problems for majority of Indonesian mathematics teachers [9]. For a long time, they are used to teaching mathematics directly from the textbook available by the government [8]. Preliminaries survey to some schools involved in the study revealed similar result. They lack of ability as well as practice to design instruction materials to pursuing mathematical reasoning skills. Besides, they also lack of knowledge and experiences in conducting lesson under scientific circumstance. As this is the case, this research is also intended to initiate and empower teachers engaged in the research to be capable and have goodwill to create mathematics classrooms as the 2013-Curriculum desires.

The 2013-Curriculum endorses teachers to implement several models of teachings; among others is problem-based learning (PBL). As a model based on constructivism paradigm, PBL leads, nurtures, and facilitates students to become problem solvers. Problem triggers such that learning take place. Students, in-group, apply their previous knowledge and experiences to solve it. By solving the problem, they construct new knowledge, grasp skills, and experience with mathematical reasoning as main tool. By reasoning, they connect information given in a problem to the database stored in their memory and draw conclusion what next should be put in place accordingly. By reasoning too, they think hardly to reveal pattern or properties contained in the problem. In addition, by reasoning they should manipulate mathematically to be able posing a generalization, a conjecture, or a proof, and explaining a mathematical idea. Doing so, students' mathematical reasoning skill will inherently improve.

In PBL, students' previous knowledge and experience form basis to handle tasks the teacher poses. Teacher should confirm that his students are ready to continue learning with adequate previous knowledge and skill. If it is not the case, he should pay attention to review essential materials supporting the continuation of learning though do it briefly.

Relating to the previous paragraph, Bieda [11] asserts 'Despite the wealth of existing literature on students' abilities to generate and understand proof, we still know very little about how skills related to justifying and proving are taught in school mathematics - particularly in mathematics courses outside of high school geometry". Furthermore, we find very few studies that examine how skills related to mathematical reasoning in general are taught at upper secondary students. Recently, reference [6] reports an effort to endorse improvement of upper secondary students' mathematical reasoning ability by means of conducting lessons based on PBL. In spite of the positive result, it took a few sample. It still needs an implementation to a wider subject. To resume, the study aims to disseminate a set of learning materials, which had been developed based on PBL in the previous study and fulfilled validity, practicality, and effectivity to inculcate upper secondary students' mathematical reasoning skills in a wider sample than before. This report constitutes result of the second of the two-years-planned research.

\section{METHODS}

The study is a developmental research. It applies the four steps Thiagarajan, Semmel, and Semmel [12] proposed. The researcher divided the four steps into two parts. First part consists of the first three steps that had been conducted last year and the last step constitutes part two of the research that took place in the second year that is in 2018. This paper reports some result the researchers got from the second year of the research. The researchers disseminate all the instruction materials that had been established valid, practice, and effective. Learning materials was taken from trigonometry topic, i.e. trigonometric comparison in four quadrants and related angels, trigonometric identity, and Sine and Cosine rules, which tenth graders should learn. Student's book plays role to help students referring necessary information in the frame of solving problem. Student's work sheet (SWS) contains problems for students to solve which expected inculcating their mathematical reasoning skills. Pre and posttest instrument were developed to measure students' MRS within four indicators, i.e. (a) Draw logical conclusion; (b) Use relationship of pattern to analyse situation, to make analogy, or to generalize; (c) Give explanation on model, fact, properties, relationship, or pattern exists; and (d) Make a conjecture and its proof.

The subjects of the research are students from four public schools; one class of each consists of 35 students averagely, with A-accreditation in Deli Serdang District, Binjai, and Medan, North Sumatera Province, Indonesia in Academic Year 2017/2018. Meanwhile, four regular teachers engaged in 
the research have varies experiences of teaching. The researcher took role an observer in the four classrooms.

Before conducting the lessons, the researcher administered pre-test to get data on previous students' MRS. Teacher then conducted the lessons, which take six times and ended by administering post-test. Both pre and post-test consist of four problems. During the lessons, the observer observe students' activity while discussing and solving problems either with or without scaffolding from their teacher. The observer recorded some parts of the students' activity. After finishing post-test, students express their perception on and assess the learning materials they have used by responding to a scale of five.

During the teaching and learning processes, the students worked in-group consisted of five or six persons solving problems in three SWS. The first SWS contained nine problems. Problems one up to five demanded the students to formulate the trigonometric comparison for related angles in every quadrant. Problem number six asked to determine which trigonometric functions have the same sign for an angle and simultaneously give the reason why. Problem number seven asked to compute the sine and cosine of an obtuse angle if the tangent is given. Problem number eight asked to compute the $\sin 50^{\circ}$ and $\tan 40^{\circ}$ in $k$ if given $\sin 150^{\circ}=k$. The last problem was the most difficult one. The teacher should play his role as a facilitator, which help the students by giving hints or clues especially to the group, who is stuck. The help could be also given classically in the classroom if most of the groups are stuck. All of the problems given are intended to facilitate the students to develop and grasp the competencies mentioned above.

SWS 2 contained six problems concerning trigonometric identities. To be able to solve the problems, students should have known some basic trigonometric identities besides algebraic manipulations. All of the problems are intended to pursuing the whole MRS indicators as well. The problems need the students to reveal the relationship pattern between the known and the task to be accomplished while applying simultaneously algebraic operation. Similarly, all of the problems contained in SWS 3 pursue the four indicators. The tasks in this SWS concern with solving problems on sine and cosine rules. One each of the problem from SWS 2 and SWS 3 are consecutively presented below.

Find the relationship between $x$ and $y$ if $x=2 \cos \theta, y=4 \sin ^{2}$ $\theta-1$.

Suppose that $\sin x+\sin y=a$ and $\cos x+\cos y=a$. Determine $\sin x+\cos x$ in $a$.

Together with the three SWS, the researcher also provided the students a book, which contained materials needed by the students to help and enable them solving the problems. The Student Book presents the related theory in brief and gives some examples but not intended to be self-contained. The researcher also provided the teacher a guided book. It contains some hints and clues for teachers to direct and lead the students solve the problems. It contains not only the hints and clues to the problems, but also the theories supporting the teaching and learning process. The teachers therefore have a grounded and firm theoretical framework in conducting the lessons.

Last, the intervention is effective if students' MRS improve at least within category low by Hake's criteria [13]. Both pre and post-test of MRS used holistic rubric for scoring 5-scale. It existed four problems, such that ideal score for each test is twenty. The score then is converted to 100 -scale.

\section{RESULTS AND DISCUSSION}

Although all of the schools involved in the study has Arank, only 15 up to $20 \%$ of them who are ready continuing learning with adequate previous knowledge as well as have intrinsic motivation to learn mathematics. Most of them were not accustomed to learning mathematics in-group, especially in constructivism environment. As this is the case, at the beginning of the lesson, teachers reviewed some important concepts, results, or principle accordingly. Furthermore, teachers also asked them to work on SWS collaboratively and each member of group be responsible of the successful of their group.

Albeit all the teachers perceived the existence of model of teaching based on constructivism, including PBL, they almost never use it to deliver the lesson nor do they ever design lesson to grasp certain high order mathematical thinking. Sometimes, they make students learning mathematics ingroup. Three of them, have engaged in training on teaching model based on scientific paradigm. Despite the training, they never design and conduct SWS themselves in pursuing mathematical reasoning skill. On the contrary, all of the teachers regard scientific approach fit for mathematics and should implement it to suitable certain topic.

One of the teachers engaged in the research did not conducted the lesson properly. She was absent for several times and it made the teaching process did not run smoothly. Besides, it is found that she hardly lead the students solving the problems in hand. She much let the students work without scaffolding. Consequently, more of the problems were left unsolved. Surely, it influenced the students' performance in the post-test. On the contrary, the other three teachers showed their efforts to lead and help the students passed the courses by solving the problems as many as possible. In fact, it was not always the case that they were able solving all the problems available.

The students' performance on the post-test is shown in Table 1. In the table, the school in the last raw is the one whose teacher was mentioned in the last paragraph. It can be seen that the students from the school performed worst. 
TABLE 1 Students Performance on MRS Test

\begin{tabular}{|l|l|l|l|l|}
\hline School & Pre-test & Post-test & N-Gain & Qualification \\
\hline SMAN A & 47.4 & 84.9 & 0.7 & Medium \\
\hline SMAN B & 10.9 & 47.3 & 0.4 & Medium \\
\hline SMAN C & 32.6 & 38.7 & 0.1 & Low \\
\hline SMAN D & 26,7 & 27 & 0 & Low \\
\hline Overall & 29.3 & 49 & 0.28 & Low \\
\hline
\end{tabular}

SMAN: Senior High School

It is seen from Table 1 that overall, the students' MRS improved. It is notably contributed by the students of the school in the first raw. Although the improvement categorized low, but two schools have their students performed better. The achievement was not surprise for during the lessons, the students of the two schools have shown progressive works. As examples, below is picked one of the problem in each SWS. In Figure 1, by the given information, the students were asked to express $\sin 50^{\circ}$ and tan $40^{\circ}$ in $k$.

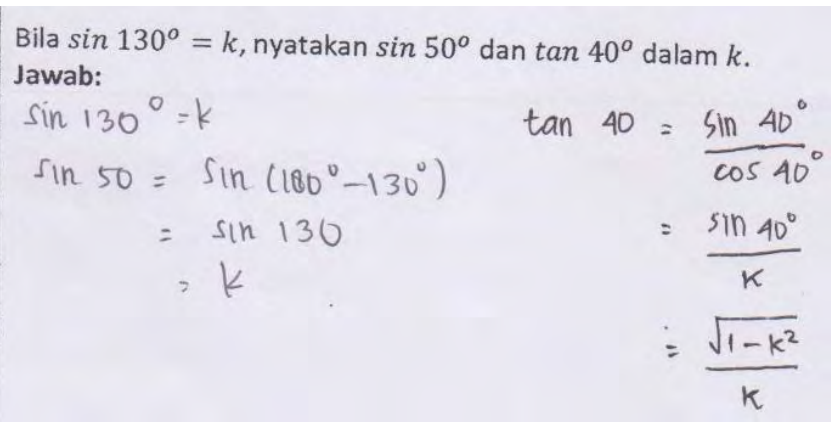

Fig. 1 Students' work on Problem number 8 of SWS 1

In Figure 1, it can be seen that the students were able to connect the given information to their previous knowledge that is the value of sine in various quadrant, especially in the second quadrant. Moreover, they also perceived the relationship between sine and cosine for complementary angle. Last, they used Pythagoras to complete the task.

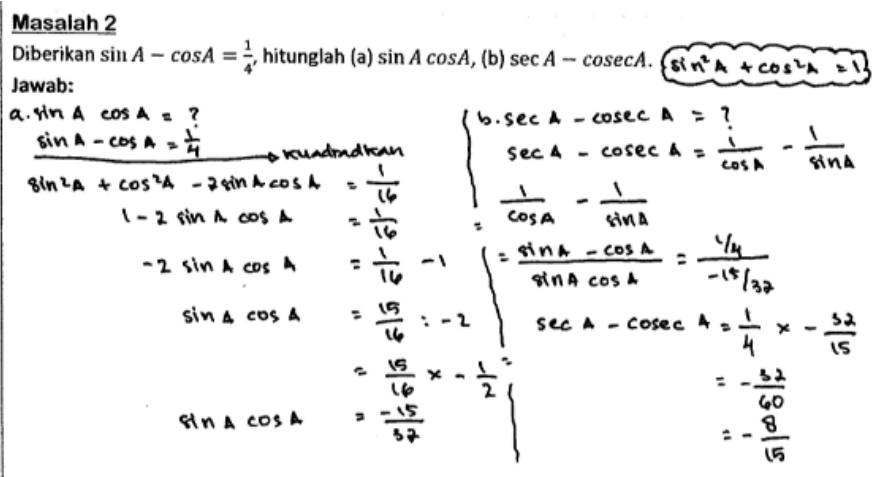

Fig. 2 Students' work on Problem number 2 of SWS 2
In Figure 2, the students are asked to compute $\sin A \cos A$ and $\sec A-\operatorname{cosec} A$ given $\sin A-\cos A=1 / 4$. From the figure, they square the equation given to produce the first demand. For the second part, they made necessary substitute and got the task completed.

In Figure 3, they are asked to determine the relationship between $x$ and $y$ given $x$ and $y$ in cosine and sine, and in tangent and secant. From the work sheet, it is seen that the students successfully finished the task correctly. They readily observe the path should be taken. That is, just square the term $x$ to get the square of cosine for on the other side they have had the term the square of sine. Doing so, what the rest to complete the tasks is to sum the two terms.

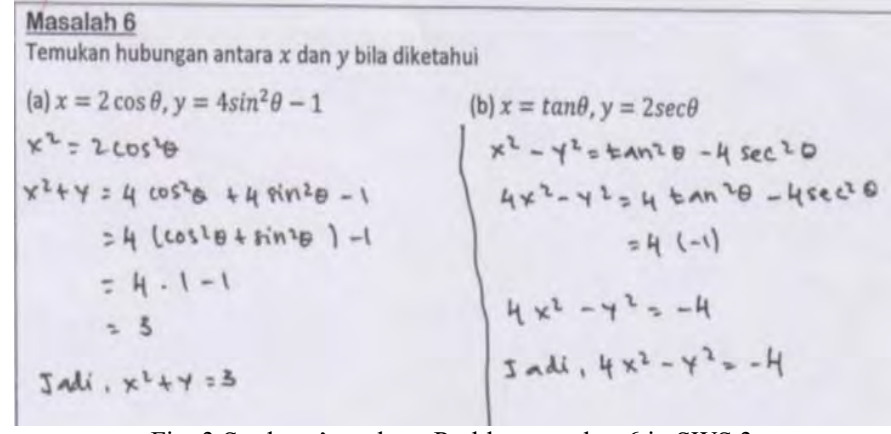

Fig. 3 Students' work on Problem number 6 in SWS 3

As had been mentioned earlier, SWS 1 contained nine problems. In the end part of the SWS, some groups were unable to complete the task or did it incorrectly. An example of students' work on problem 9 is shown in Fig. 4a and Fig. $4 \mathrm{~b}$. Given the length of $B C$ equals 1 , it asks to determine the length of $B D, A D, F B, A E$, and $D E$, sin $75^{\circ}, \cos 75^{\circ}$, and tan $75^{\circ}$ (see Fig. 4)

From Fig. 4a, which exhibit the students' work, they correctly calculate the length of $B D, F B$, and $A D$. While doing this, they had in mind some minor works. For example, they had in mind that the length of $A B$ is $\sqrt{ } 2$ for the triangle $A B C$ is an isosceles one with the length of its leg is unit. They then continue working on calculating the length of $A E$ (Fig. 4b). However, they committed mistake when calculating the length of $D E$. Instead of using Pythagorean (executed incorrectly), it will give more advantage by observing that $D E=D F+F E$ and then compute the length of $D F$ via triangle $B D F$.

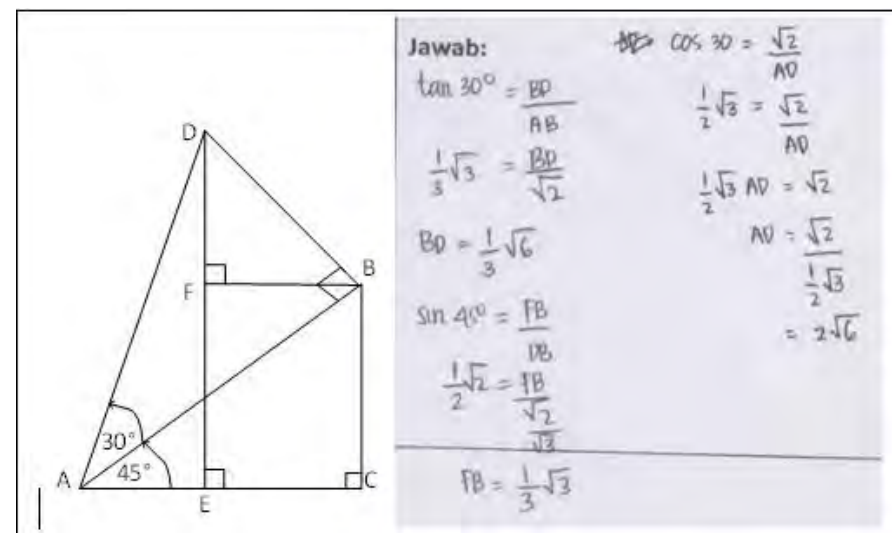




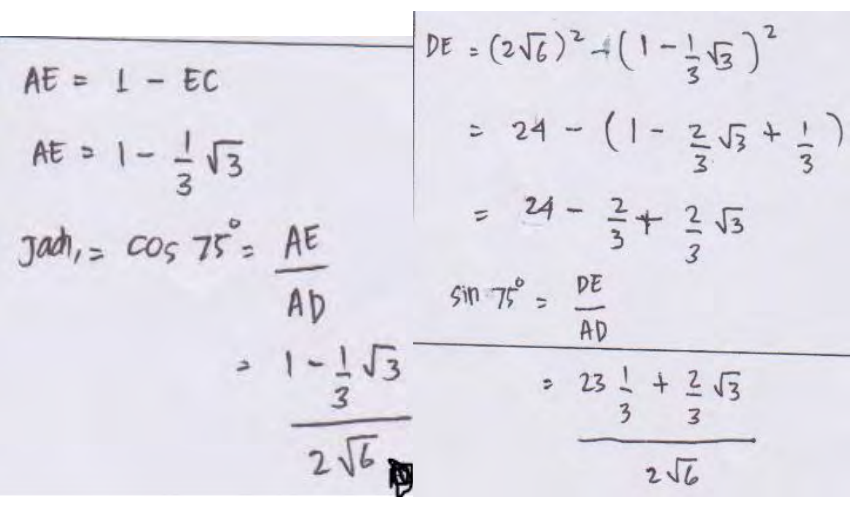

Fig. 4b Students' work on problem 9

For the mistake, they then continued to another mistake accordingly, that is the value of $\sin 75^{\circ}$. Finally, they did not calculate $\tan 75^{\circ}$. This work was the best performance for problem number nine in SWS 1. The other groups worked worse.

SWS 2 contained six problems, which is intended to inculcate students' skill in all the four indicators mentioned earlier. All of the groups failed in problem number 6. Besides, no group worked on the second part of problem number 5 correctly, which demand student's skill in the first and the second indicator. The problem demands the students to determine $\operatorname{cosec} x-\cot x$ and $\cos x$ while given $\operatorname{cosec} x+\cot x$ $=3$. Figure 5 exhibit the work of one group on problem number 5. From the figure, it looks that the students confused about addition and multiplication of fraction and therefore again committed mistake.

$$
\begin{aligned}
& \frac{1+\cos x}{1-\cos ^{2} x}=3
\end{aligned}
$$

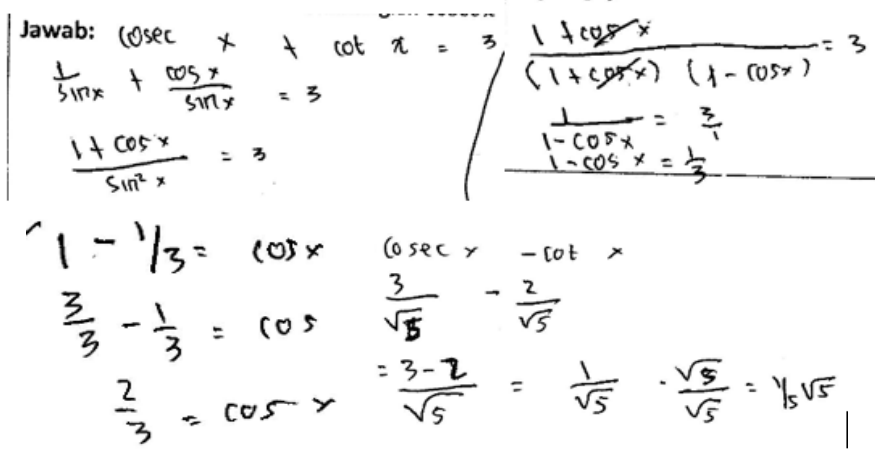

Fig. 5 Students' work on problem 5 of SWS 2

In this case, the students should observe the known and the demand and then use relationship between cosecant and cotangent to operate algebraically. Of course, the task become easy if they perceived to multiply the known with its conjugate. Problem number six demands the students create a relationship between the given. The majority of the students were not able to complete this task. It is very difficult for them to reveal what and how to do to approach the solution. In fact, with the basic knowledge on trigonometry they have, it is possibly to make trial and gradually arrive at the target. Some of them committed incorrect operation algebraically as Figure 6 shows. The fault is not responsible to trigonometry, but algebra. They did not perceive that $4 \cos ^{2} \theta=x^{2}$. In the second part of this problem, they repeated the fault. Albeit the fault, they really made a significant progression, for they were able to observe the relationship between the given to the target and ran a suitable algebraic operation to approach the solutions.

\begin{tabular}{|c|c|c|}
\hline & \multicolumn{2}{|c|}{ (b) $x=\tan \theta, y=2 \sec \theta$} \\
\hline (a) $\because=2 \cos \theta, y=4 \sin$ & 1 b) $x=\tan \theta$ & $\left(1 / 4 y^{2}=\sec ^{2} \theta\right.$ \\
\hline a) $\begin{array}{l}x=2 \cos \theta \\
y=4 \sin ^{2} \theta-1\end{array}$ & $\begin{array}{c}y=2 \sec \theta \\
x^{2}=(\tan \theta) 2\end{array}$ & $x^{2}-y_{4} y^{2}+1=0$ \\
\hline$y=4\left(1-\cos ^{2} \theta\right)-1$ & $\alpha^{2}=\tan ^{2} \theta$ & \\
\hline$f=4-4 \cos ^{2} \theta-1$ & $\begin{array}{l}x^{2}=\sec ^{2} \theta-1 \\
x^{2}=y y^{2}-1\end{array}$ & \\
\hline $\begin{array}{l}y=3-4 \cos ^{2} \theta \\
y=3-4 x\end{array}$ & $y^{2}=(2 \sec \theta)^{2}$ & \\
\hline$y=3-4 x$ & $y^{2}=\Delta \sec ^{2} \theta$ & \\
\hline
\end{tabular}

Fig. 6 Students' work on problem 6 of SWS 2

The last SWS contained five problems. Similar to SWS 2, all of the problems in SWS 3 pursuit the four indicators mentioned above. All tasks are based on sine rule and trigonometric identities. Most groups were able to complete the tasks but problem 4. In this task, given $\sin x+\sin y=a$ and $\cos x+\cos y=a$. It demands to determine $\sin x+\cos x$ in $a$. This task inculcate students' skills especially in the first, the second and third indicators. It needs multiple steps to complete the task, the ability to create new equation from old ones, operate equation in hand to lead to tentative result, and draw conclusion by connecting the result to previous ones. Instead of doing this, the students only wrote something nonsense (see Fig. 7). Probably, of all the problems presented in the teaching and learning process, this is the most difficult one for the students.

$$
\begin{aligned}
& \text { Misalkan } \sin x+\sin y=a \text { dan } \cos x+\cos y=a \text {. Tentukan } \sin x+\cos x \text { dalam } a . \\
& \text { Jawab: } \\
& =\sin x+\sin y+\cos x+\cos y=2 a . \\
& \quad t(\sin x+\cos x)+(\sin y+\cos y)=2 a . \\
& =\left(\frac{1}{2} a+\frac{1}{2} a\right)+\left(\frac{1}{2} a+\frac{1}{2} a\right)=2 a . \\
& a+a=2 a .
\end{aligned}
$$

\section{Maka: $\sin x+\cos x=a$.}

Fig. 7 Students' work on problem 4 of SWS 3

Overall, considering their work on the three SWS, the researcher conclude there exists progress in students' mathematical reasoning skills. Concerning to the SWS, on general, they perceived the problems were difficult, though making sense and believed that they would be able to solve them if given some help. Moreover, they admitted the SWS 
enforced them to work more actively than ever and enhanced their skills in problem solving.

The low improvement occurred in the research, surely due to many causes. Initial data had revealed that the students were not accustomed to learning in scientific approach environment. Even though, they ever learned in-group, but it was not in the frame of solving problems. Moreover, it is a certainly challenge conducting a PBL lesson in a mathematical classroom with only 15 up to $20 \%$ of students ready continue learning by means of having adequate previous knowledge [6]. They even resist on the approach such as PBL. Most of them demanded teacher to teach as usual, that is explain the theory then give examples and continue working on problems similar to examples. That is why they wished the students' book (SB) contained more explanation and more worked-problems. Actually, the SB was designed as supplement to textbook they used.

Theoretically, PBL demands teachers keeping the learning environment in which students engage in high-level thinking and reasoning. For lack of experience in conducting lesson using teaching model such as PBL, the teachers were not able scaffolding fluently. Consequently, it occurred time passed by inefficiently, which also stressed in [11].

The lack of scaffolding was also noted in the observation sheet on students' activity. Emerged neither different idea or any suggestion, nor any effort to work in different way or pose question that need deep explanation in part of the students. All of those high-level thinking or reasoning will emerge in case teacher pose varies, connecting and challenging questions.

Based on experiences, the teachers said trigonometry is one of the most difficult topic for students to handle. It contains too many identities, possibly relationships among others, possibilities to develop new relation, and connections to geometry which also difficult for them. It is therefore, more of the students have less intention, perseverance, and passion to work on it. It certainly contribute to the weakness of students' mathematical reasoning skills [6].

\section{CONCLUSION}

The research showed that the dissemination of the instructional devices which based on problem-based learning successfully improved the students' MRS. As a model of teaching, PBL is concluded reliable to inculcating students' MRS. It is therefore suggested the teacher to applying the device in their classroom especially while pursuing the goals aforementioned. To grasp more successfulness, it is preferable the teacher who conduct the lesson master the teaching model used and be able to apply it fluently.

\section{ACKNOWLEDGMENT}

This research was supported by the Ministry of Research, Technology and Higher Education of the Republic of Indonesia. The authors are thankful to Rector of State University of Medan, Research Director, and Dean of Faculty of Mathematics and Natural Sciences for providing necessary facilities to conduct the research.

\section{REFERENCES}

[1] M. Henningsen and M. K. Stein, "Mathematical Tasks and Student Cognition: Classroom-Based Factors That Support and Inhibit HighLevel Mathematical Thinking and Reasoning". Journal for Research in Mathematics Education, Vol. 28, No. 5, 524-549, 1997.

[2] National Council of Teachers of Mathematics, "Curriculum and Evaluation Standards for School Mathematics". Reston VA: NCTM, 1989.

[3] J. Lithner, "Principles for designing mathematical tasks that enhance imitative and creative reasoning". ZDM Mathematics Education, DOI 10.1007/s11858-017-0867-3, 2017.

[4] I. V. S. Mullis, M. O. Martin, P. Foy, A. Arora, "TIMSS 2011 International Result in Mathematics". Boston, MA: TIMSS and PIRLS International Study Center, 2012.

[5] I. V. S. Mullis, M. O. Martin, P. Foy, M. Hooper, "TIMSS 2015 International Result in Mathematics". Chestnut Hill, MA: TIMSS and PIRLS International Study Center, 2016.

[6] E. E. Napitupulu, "On Developing Instruction Materials in Inculcating Secondary Students' Mathematical Reasoning Skills". Advances in Social Science, Education and Humanities Research, Atlantis Press, Vol. 104, No. 104, pp. 174-178, 2017.

[7] E. E. Napitupulu, D. Suryadi, Y. S. Kusumah, "Cultivating Upper Secondary Students' Mathematical Reasoning Ability And Attitude Towards Mathematics Through Problem-Based Learning". Journal on Mathematics Education, Vol. 7, No. 2, pp. 61-71, 2016.

[8] F. Shadiq, "Laporan Hasil Seminar dan Lokakarya Pembelajaran Matematika" Yogyakarta: P4TK (PPPG) Matematika, 2007.

[9] F. Jalal, M. Samani, M. C. Chang, R. Stevenson, A. B. Bagatz, S. D. Negara, "Teacher Certification in Indonesia: A Strategy for Teacher Quality Improvement". Indonesia: Department Pendidikan Nasional Indonesia, 2009.

[10] Indonesian Ministry of Education and Culture, "Kurikulum 2013". Jakarta: Kemendikbud, 2013.

[11] K. N. Bieda, "Enacting Proof-Related Tasks in Middle School Mathematics: Challenges and Opportunities". Journal for Research in Mathematics Education, Vol. 41, No. 4, 351-382, 2010.

[12] S. Thiagarajan, D.S.Semmel, \&M.Semmel, "Instructional Development for Training Teachers of Exceptional Children. A Sourse Book". Blomington: Central for Innovation on Teaching the Handicapped, 1974.

[13] R. R. Hake, "Interactive-Engagement versus Traditional Methods: A Six-Thousand-Student Survey of Mechanics Test Data for Introductory Physics Courses". Am. J. Phys., 66 (1), P. 65, 1998. 